\title{
Correlation Between Match Performance and Field Tests in Professional Soccer Players
}

\author{
by \\ Paulo E. Redkva', Mauro R. Paes², Ricardo Fernandez², Sergio G. da-Silva ${ }^{1}$
}

The aim of this study was to investigate possible correlations between aerobic and anaerobic fitness (in field tests) with performance during a 90 min friendly match, through the following variables: total distance covered, maximal running speed, number of high-intensity actions and number of sprints. Eighteen professional soccer players from a Brazilian elite team (age $23 \pm 3$ years, body mass $77.5 \pm 8.9 \mathrm{~kg}$ ) participated in the study. The athletes performed a Yo-Yo Endurance Test (aerobic fitness) and a Running Anaerobic Sprint Test (six maximal $35 \mathrm{~m}$ efforts separated by $10 \mathrm{~s}$ of passive recovery, anaerobic fitness). Data were collected during friendly matches using a GPS with $5 \mathrm{~Hz}$ technology. To establish the correlation between the variables determined during the matches, the Pearson correlation coefficient was used (significance level of $p \leq 0.05$ ). A high correlation was found between distance covered in the Yo-Yo endurance test and total distance covered $(r=0.72 ; p<0.05)$, number of high-intensity actions $(r=0.78 ; p<0.05)$ and number of sprints $(r=0.88 ; p<0.01)$ in the soccer matches. The RAST variables did not relate to the standards set during the matches $(p<0.05)$. From the results of the present study, it may be concluded that there is no correlation between RAST and friendly match data variables. However, better results in the Yo-Yo endurance test correlate with total distance, number of high-intensity actions and sprints during matches.

Key words: Yo-Yo Endurance Test, RAST, soccer evaluation, GPS.

\section{Introduction}

Soccer is a complex sport (Casanova et al., 2013), and to play this game at a competitive level, players require a high level of aerobic (i.e., mitochondrial metabolic pathways) and anaerobic (i.e., non-mitochondrial metabolic pathways) fitness (Reilly et al., 2000). The aerobic fitness level (i.e., maximal oxygen uptake - $\mathrm{VO}_{2 \mathrm{MAx}}$ ) in professional soccer players is associated with distance covered at high-intensity during a match (Bangsbo, 1994). Several studies have revealed total distance covered to be about $10-12 \mathrm{~km}$ at a relative intensity of $75 \%$ of $\mathrm{VO}_{2 \mathrm{MAX}}$ (Bangsbo, 1994; Helgerud et al., 2001; McMillan et al., 2005), consequently contributing about $90 \%$ of the total energy cost of the game (Bangsbo, 1994; Helgerud et al., 2001). High-level aerobic demand in soccer is essential to provide fast recovery after a high- intensity activity (Bishop et al., 2004; Helgerud et al., 2001; McMillan et al., 2005).

Although the energy pathway during a soccer match is provided principally by the aerobic metabolism, the anaerobic pathway is called upon during high-intensity actions such as jumping, kicking, and even movements to mark the opponent (Bangsbo, 1994; Di Salvo et al., 2009; Krustrup et al., 2003; Stolen et al., 2005). The contributions of non-mitochondrial metabolic pathways (phosphagen and glycolytic energy pathways) during a match have been evidenced by the high level of peak blood lactate $(\sim 10$ $\left.\mathrm{mmol} \cdot \mathrm{L}^{-1}\right)$, followed by a decrease in muscle $\mathrm{pH}$ ( 6.8) (Bangsbo at al., 2007), distance covered at high-intensity $(1.1 \mathrm{~km}$ covered at a speed above 18 $\mathrm{km} \cdot \mathrm{h}^{-1}$ ) (Burgess et al., 2006) and the number of

1 - Research Center on Exercise and Sports, Department of Physical Education, Federal University of Parana, Brazil.

2 - Department of Physiology, Federal University of Parana, Brazil. 
intense actions during the game (between 150 and 250) (Bangsbo et al., 2007). Moreover, activities such as sprints represent about $8-12 \%$ of the total distance covered during the game (Rampinini et al., 2007). Thus, repeated-sprint ability (RSA) with short rest intervals appears to be a valid indicator of player's performance, being considered fundamental for sports events with important anaerobic metabolic demand (Bishop et al., 2011).

Due to the importance of both the aerobic and anaerobic energy systems in soccer, many tests have been used to monitor and prescribe training during the competitive season (Chamari et al., 2005). Additionally, the strategy of evaluating physical performance in friendly matches with a global positioning system (GPS) is currently a common procedure employed by sports scientists and performance analysts in elite soccer (Mallo et al., 2015). The physical demands imposed on elite players have been extensively documented in recent years (Bradley et al., 2009; Dellal et al., 2011; Di Salvo et al., 2007; Rampinini et al., 2007). The short time that teams have to carry out physiological assessments, as well as the high financial cost of testing in controlled environments, should be considered (Svensson and Drust, 2005). There is a number of studies focused on identifying the physiological characteristics of players from different levels. However, only few papers can effectively relate the results obtained in field tests with variables correlated to physical demands during the game.

Therefore, the aim of this study was to verify possible relationships between the distance covered, maximal running speed and number of high-intensity actions performed during a friendly soccer match with aerobic and anaerobic fitness in professional players.

\section{Methods}

\section{Participants}

The study included 18 professional soccer players (age $23 \pm 3$ years, body mass $77.5 \pm 8.9 \mathrm{~kg}$, body height $177 \pm 5 \mathrm{~cm}$, percentage body fat mass $11.5 \pm 1.5 \%, \quad \mathrm{VO}_{2 \mathrm{MAX}} 56.8 \pm 3.9 \mathrm{~mL} \cdot \mathrm{kg}^{-1} \cdot \mathrm{min}^{-1}$ ), belonging to the first division soccer team of the state of Paraná, Brazil. Data collection was performed during the 2014 pre-season. These athletes had been engaged in regular training and competitions for at least 5 years and performed approximately 10 training sessions per week with average duration of $2 \mathrm{~h}$ each. In the study only athletes who had no osteomyoarticular injuries and who were not taking anti-inflammatory drugs were included. During the period of the study, no player was injured. All procedures complied with the declaration of Helsinki regarding human experimentation and were approved by the local Scientific and Ethics Committee. All players were informed of the procedures, including the risks associated with participation in the study, and written informed consent was obtained.

\section{Design and Procedures}

Initially, body composition of the participants was evaluated. Following this, all tests were performed on a soccer field. The players performed a Yo-Yo Endurance test (aerobic fitness) and the running anaerobic sprint test (RAST) for determination of anaerobic fitness (separated by $24-48 \mathrm{~h}$ ). Players completed the tests on an official natural grass field, wearing cleats that met the standards required by the sport.

After the initial assessments, data were collected during four friendly matches, according to a schedule established by the authorities between teams of the same level of the Paranaense Football Federation using a GPS (QStarz ${ }^{\circledR}$, Taipei, Taiwan) with $5 \mathrm{~Hz}$ technology (Varley et al., 2012).

\section{Body composition}

For the measurement of body mass, a professional scale was used with precision of 100 g (Filizola, São Paulo, Brazil) and to determine body height, a stadiometer (Sanny ${ }^{\circledR}$ ) with accuracy to the nearest $0.1 \mathrm{~cm}$. Skinfold thicknesses were measured using a caliper to the nearest $0.1 \mathrm{~mm}$ (Cescorf ${ }^{\circledR}$, Porto Alegre, Brazil). The Faulkner equation was used to calculate body fat percentage: \% Body Fat $=5.783+0.153 \times(\mathrm{SE}+$ $\mathrm{TR}+\mathrm{SI}+\mathrm{AB}$ ); where $\mathrm{SE}=$ subscapular, $\mathrm{TR}=$ triceps, $\mathrm{SI}=$ suprailiac, $\mathrm{AB}=$ abdominal.

\section{Yo-Yo Endurance Test (YET)}

The YET (continuous) is a variation of the Yo-Yo test series (Bangsbo, 1994) consisting of repeated $2 \times 20 \mathrm{~m}$ runs back and forth between the starting, turning, and finishing lines at a progressively increased speed controlled by audio beeps from a tape recorder. When the participant stops, the final speed and the number of performed $20 \mathrm{~m}$ distances at this speed are recorded, including the final run. The test data 
were inserted into AVAesporte ${ }^{\circledR}$ software (Sports Systems, MG. Brazil) to obtain the distancecovered values for each athlete. Players performed the test on an official natural grass field, wearing cleats that met the standards required by the sport.

\section{Running Anaerobic Sprint Test (RAST)}

The RAST (Zagatto et al., 2009), consisting of six maximal $35 \mathrm{~m}$ all out sprints, interspaced by $10 \mathrm{~s}$ of passive recovery, was used for determination of anaerobic fitness. During the test, running time was measured using a photocell system (Cefise ${ }^{\circledR}$, São Paulo, Brazil) located at the start and end of the $35 \mathrm{~m}$ track. Prior to the start of the test, the players were weighed, wearing clothes, to determine total body weight. These variables were utilized to calculate power.

\section{Power(W)=[weight $\left.(\mathrm{kg}) \times \operatorname{distance}^{2}(\mathrm{~m})\right] / \operatorname{time}^{3}(\mathrm{~s})$}

Thus, the peak power (PP, the highest power value of the six efforts of $35 \mathrm{~m}$ ), mean power (MP, the mean power value of the six efforts of $35 \mathrm{~m}$ ) and minimum power (MinP, the smallest power value of the six efforts of $35 \mathrm{~m}$ ) were obtained. The same RAST variables were recorded relative to the athletes' body weight (i.e., $\mathrm{PPR}=\mathrm{PP} /$ weight; $\mathrm{MPR}=\mathrm{MP} /$ weight). The fatigue index [FI (\%)] of the RAST was obtained through the following equation:

$$
\text { FI }(\%)=(P P-M i n P) \times 100 / P P
$$

\section{Match Analysis}

The friendly matches used for data collection were carried out in Jan/2014, following the same standards as used in official matches, with two periods of $45 \mathrm{~min}$ and a $15 \mathrm{~min}$ half time interval. The matches took place at $16 \mathrm{~h}$.

Before each match, the players performed a standardized, specific warm-up, typically used in official games. The two evaluated teams were organized tactically, playing the 4-4-2 system. The GPS was positioned on the player's arm, according to the manufacturer's recommendations. All players on the team, except the goalkeeper, received the equipment at the start of the match. There was an agreement between the technicians/coaches to create opportunities for a larger number of substitutions than generally allowed in the rules of soccer, thus, only the data from players who completed the friendly match, playing both halves of $45 \mathrm{~min}$ without being replaced were considered.

For the analysis of motor actions during the soccer game with the GPS, such variables were identified as total distance covered, maximal running speed, high-intensity actions (running) and number of sprints. The criteria for classification of high-intensity sprints and actions were adapted from Aughey and Varley (2013) and the data were analyzed in Qsports ${ }^{\circledR}$ software (QStarz ${ }^{\circledR}$, Taipei, Taiwan). The values adopted were: 1) high-intensity actions (running): actions in which the player reached a speed between 15.9 and $24 \mathrm{~km} \cdot \mathrm{h}^{-1}$; 2) sprints: actions in which the player reached a speed above $24 \mathrm{~km} \cdot \mathrm{h}^{-1}$.

\section{Statistical Analysis}

Results are presented as mean \pm standard deviation and confidence intervals of $95 \%$ (95\% CI). Initially, the Shapiro-Wilk test was used to confirm the data normality $(\mathrm{n}<50)$. To verify the correlation between the results obtained in the YET and RAST with the variables of the soccer matches in the study (total distance covered, maximal running speed, high-intensity actions (running) and number of sprints), the Pearson correlation was used. The correlations were distributed according to R-values, which were classified as very weak ( 0.0 to 0.2$)$, weak $(0.2$ to $0.4)$, moderate (0.4 to 0.7$)$, strong (0.7 to 0.9$)$ and very strong (0.9 to 1.0) (Rowntree, 1981). In all cases a significance level of $5 \%(p<0.05)$ was assumed. All data were analyzed using the software package SPSS Statistics 19.0 (SPSS Inc., Chicago, IL, USA).

\section{Results}

Individual data of total distance covered, maximal running speed, high-intensity actions (running) and number of sprints in the friendly matches are presented in Table 1.

The distance covered and the maximal velocity corresponding to the YET were $2027 \pm 215$ $\mathrm{m}(95 \%$ CI $1.382-1.510 \mathrm{~m})$ and $13.0 \pm 0.5 \mathrm{~km} \cdot \mathrm{h}^{-1}$ (95\% CI $\left.13.7-14.3 \mathrm{~km} \cdot \mathrm{h}^{-1}\right)$, respectively. Significant correlations were found between the distance covered $(\mathrm{m})$ in the YET with total distance covered, high-intensity actions (running) and number of sprints performed in the friendly matches (Table 2).

The mean PP, MP and FI (RAST variables) 
are presented in Table 3. No significant correlations were found between the performance variables obtained during the matches and RAST variables (Table 3 ).

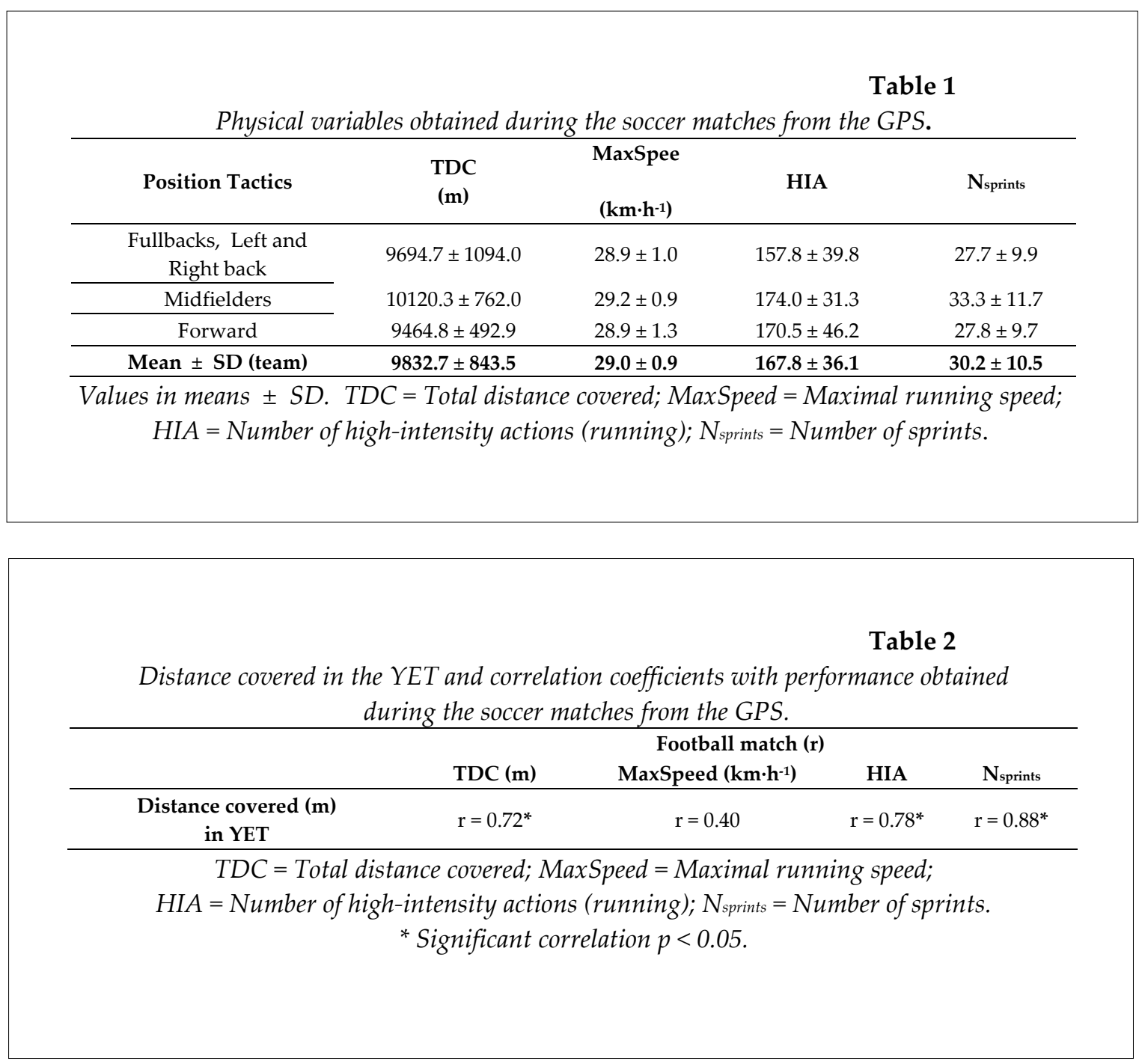

Table 3

RAST variables and correlation coefficients with performance in the soccer matches.

\begin{tabular}{|c|c|c|c|c|c|}
\hline \multirow[b]{2}{*}{ RAST } & \multirow[b]{2}{*}{ Mean \pm SD $($ CI95\%) } & \multicolumn{4}{|c|}{ Football match (r) } \\
\hline & & DC & axSpeed & HIA & $\mathbf{N}_{\text {sprints }}$ \\
\hline PP (W) & $933.5 \pm 118.1(874.7-992.1)$ & $\mathrm{r}-0.22$ & r 0.44 & r 0.01 & r -0.05 \\
\hline PP $\left(W \cdot k^{-1}\right)$ & $12.0 \pm 0.8(11.5-12.4)$ & r -0.40 & $\mathrm{r} \quad-0.18$ & $\mathrm{r}-0.26$ & r -0.23 \\
\hline MP (W) & $761.5 \pm 92.4(715.6-807.5)$ & r -0.18 & r 0.45 & r -0.11 & $\mathrm{r}-0.05$ \\
\hline $\mathrm{MP}\left(\mathrm{W} \cdot \mathrm{kg}^{-1}\right)$ & $9.4 \pm 0.7(9.4-10.1)$ & $\mathrm{r}-0.32$ & r -0.20 & $\mathrm{r} \quad-0.05$ & $\mathrm{r}-0.21$ \\
\hline FI (\%) & $33.2 \pm 5.91(30.3-36.2)$ & r 0.66 & $\mathrm{r}-0.11$ & $\mathrm{r}-0.11$ & r -0.07 \\
\hline
\end{tabular}

RAST = Running Anaerobic Sprint Test $;$ TDC = Total distance covered;

MaxSpeed = Maximal running speed;

$H I A=$ Number of high-intensity actions (running);

$N_{\text {sprints }}=$ Number of sprints. ${ }^{*}$ Significant correlation $p<0.05$. 


\section{Discussion}

The main finding of this study was a high correlation between the total distances covered during the aerobic fitness test (YET) and performance during the friendly matches (Table 3). However, no significant correlation was found between performances in the anaerobic fitness test (RAST) with the variables studied during the same match.

As can be seen in Table 3, distance covered during the YET was strongly correlated with most of the performance variables registered during the matches, except maximal running speed $(r=0.40 ; p=0.23)$. In modern soccer, the aerobic system plays an important role in the player's performance, and this metabolic pathway is the predominant supplier of energy during most of the match (Bangsbo, 1994; Helgerud et al., 2001) and enables rapid recovery of the anaerobic system after high intensity efforts (Bishop et al., 2004; Helgerud et al., 2001; McMillan et al., 2005). The literature describes non-invasive procedures that estimate aerobic fitness of players such as the YET (Krustrup et al., 2003). Metaxas et al. (2005), suggested that professionals involved in the assessment of physical fitness of soccer players used the YET for assessing aerobic capacity throughout the season. It was observed that athletes with better performance in this type of a test could be more effective during a match (Castagna et al., 2006). Furthermore, its applicability is satisfactory, since it is possible to evaluate several athletes simultaneously and it does not require sophisticated equipment being thus low-cost (Fornaziero et al., 2009). In the present study, the high correlation (Table 3) indicates that distance covered in the YET to a large degree explains performance (variables observed) in the friendly soccer matches.

Surprisingly, no correlations were observed between the RAST variables (PP, MP and FI), which could also be considered a repeated sprint ability test (Beck et al., 2014), and total distance covered, maximal running speed, high-intensity actions (running) and number of sprints during the friendly matches (Table 4). A possible explanation for the lack of correlations may be related to the difference between time of efforts, distance and recovery intervals present in the RAST, being different from the actions presented by the players in the match situation.
As reported by Dwyer and Gabbett (2012) the majority of sprints on sports fields last around 1-2 s, usually with speed values ranging above 21 $\mathrm{km} \cdot \mathrm{h}^{-1}$. The average sprint distance in soccer players presented by the authors was $5.8 \mathrm{~m}$, with peaks of maximum speed of around 29 km.h-1 (Dwyer and Gabbett, 2012). Carling et al. (2012), in a study with professional players, showed high-intensity distances of $16 \pm 4.9 \mathrm{~m}$ and $2.7 \pm 0.7$ s. Bradley et al. (2009) demonstrated that a player performed actions with an intensity above 19.8 km.h-1 every 72 s. As the RAST uses an intermittent effort for the achievement of maximum efforts $(\sim 5 \mathrm{~s})$, contribution of the aerobic metabolism (Milioni et al., 2016) in the intervals between the high intensity efforts and sprints could play an important role in maintaining performance, such as removing intracellular inorganic phosphate $(\mathrm{Pi})$ and the resynthesis of phosphocreatine stores (PCr) (Kalva-Filho et al., 2013). In addition, when maximum efforts are repeated with short rest intervals, the aerobic contribution increases during the exercise and can be decisive in maintaining high intensity performance (Girard et al., 2011; Milioni et al., 2016). Thus, it seems possible that the best performance indices in high intensity intermittent efforts are dependent on aerobic fitness (Bishop and Edge, 2006). This could be due to the RAST consisting of forward sprints, without changes of direction, which little resemble efforts in soccer. Furthermore, the actions in the game could be sustained by the contribution of the aerobic metabolism (i.e., a high relationship between aerobic fitness and actions in the match).

Practical implications based on our results may be related to the important focus that must be given to conditioning to develop the aerobic metabolism, since this physical capacity seems to be fundamental in the execution of actions of high physical demands during the games of soccer. Also, the use of the RAST as a predictor of high intensity actions, number of sprints and maximum speed developed should be treated with caution.

We concluded that professional soccer players with better results in the YET covered a greater total distance and performed a larger number of high-intensity actions and sprints 
during the matches. On the other hand, the results of the anaerobic indices (through the RAST) did not correlate with performance during the matches.

\section{Acknowledgements}

The authors would like to thank soccer players for their kind participation and coaches for the opportunity to investigate.

\section{References}

Bangsbo J. Energy demands in competitive soccer. J Sport Sci, 1994; 12: S5-12

Bangsbo J, Iaia FM, Krustrup P. Metabolic response and fatigue in soccer. J Sports Physiol Perform, 2007; 2(2): 111

Beck WR, Zagatto AM, Gobatto CA. Repeated sprint ability tests and intensity-time curvature constant to predict short-distance running performances. Sport Sci Health, 2014; 10(2): 105-110

Bishop D, Edge J. Determinants of repeated-sprint ability in females matched for single-sprint performance. Eur J Appl Physiol, 2006; 97(4): 373-379

Bishop D, Edge J, Goodman C. Muscle buffer capacity and aerobic fitness are associated with repeated-sprint ability in women. Eur J Appl Physiol, 2004; 92(4-5): 540-547

Bishop D, Girard O, Mendez-Villanueva A. Repeated-sprint ability-Part II. Sports Med, 2011; 41(9): 741-756

Bradley PS, Sheldon W, Wooster B, Olsen P, Boanas P, Krustrup P. High-intensity running in English FA Premier League soccer matches. J Sport Sci, 2009; 27(2): 159-168

Burgess D, Naughton G, Norton K. Profile of movement demands of national football players in Australia. J Sci Med Sports, 2006; 9(4): 334-341

Carling C, Le Gall F, Dupont G. Analysis of repeated high-intensity running performance in professional soccer. J Sport Sci, 2012; 30(4): 325-336

Casanova F, Garganta J, Silva G, Alves A, Oliveira J, Williams AM. Effects of prolonged intermittent exercise on perceptual-cognitive processes. Med Sci Sports Exerc, 2013; 45(8): 1610-1617

Castagna C, Impellizzeri FM, Belardinelli R, Abt G, Coutts A, Chamari K, D'Ottavio S. Cardiorespiratory responses to Yo-yo Intermittent Endurance Test in nonelite youth soccer players. J Strength Cond Res, 2006; 20(2): 326-330

Chamari K, Hachana Y, Kaouech F, Jeddi R, Moussa-Chamari I, Wisløff U. Endurance training and testing with the ball in young elite soccer players. Br J Sports Med, 2005; 39(1): 24-28

Dellal A, Chamari K, Wong DP, Ahmaidi S, Keller D, Barros R, Carling C. Comparison of physical and technical performance in European soccer match-play: FA Premier League and La Liga. Eur J Sport Sci, 2011; 11(1): 51-59

Di Salvo V, Baron R, Tschan H, Montero FC, Bachl N, Pigozzi F. Performance characteristics according to playing position in elite soccer. Int J Sport Med, 2007; 28(3): 222

Di Salvo V, Gregson W, Atkinson G, Tordoff P, Drust B. Analysis of high intensity activity in Premier League soccer. Int J Sport Med, 2009; 30(3): 205-212

Dwyer DB, Gabbett TJ. Global positioning system data analysis: Velocity ranges and a new definition of sprinting for field sport athletes. J Strength Cond Res, 2012; 26(3): 818-824

Fornaziero AM, Leite R, De Azevedo P, Dourado A, Daros L, Osiecki R, Stanganelli L. Comparative analysis of the performance of soccer players between two aerobic power tests: treadmill and Yo-Yo intermittent endurance test level 2. Rev Andaluza Med Deporte, 2009; 2(3): 82-86

Girard O, Mendez-Villanueva A, Bishop D. Repeated-sprint ability-Part I. Sports Med, 2011; 41(8): 673-694

Helgerud J, Engen LC, Wisloff U, Hoff J. Aerobic endurance training improves soccer performance. Med Sci Sports Exerc, 2011; 33(11): 1925-1931 
Kalva-Filho CA, Loures JP, Franco VH, Kaminagakura EI, Zagatto AM, Papoti M. Relationship between aerobic parameters and intermittent high-intensity effort performance. Motriz, 2003; 19(2): 306-312

Krustrup P, Mohr M, Amstrup T, Rysgaard T, Johansen J, Steensberg A, Bangsbo J. The yo-yo intermittent recovery test: physiological response, reliability, and validity. Med Sci Sports Exerc, 2003; 35(4): 697-705

Mallo J, Mena E, Nevado F, Paredes V. Physical Demands of Top-Class Soccer Friendly Matches in Relation to a Playing Position Using Global Positioning System Technology. J Hum Kinet, 2015; 47(1): 179-188

McMillan K, Helgerud J, Grant S, Newell J, Wilson J, McDonald R, Hoff J. Lactate threshold responses to a season of professional British youth soccer. Br J Sports Med, 2005; 39(7): 432-436

Metaxas TI, Koutlianos NA, Kouidi EJ, Deligiannis AP. Comparative study of field and laboratory tests for the evaluation of aerobic capacity in soccer players. J Strength Cond Res, 2005; 19(1): 79-84

Milioni F, Zagatto AM, Barbieri RA, Andrade VL, Dos-Santos JW, Gobatto CA, Papoti M. Energy systems contribuition in the running-based anaerobic sprint test. Int J Sport Med, 2017; 38(3): 226-232

Rampinini E, Coutts A, Castagna C, Sassi R, Impellizzeri F. Variation in top level soccer match performance. Int J Sport Med, 2007; 28(12): 1018-1024

Reilly T, Bangsbo, Franks A. Anthropometric and physiological predispositions for elite soccer. J Sport Sci, 2000; 18(9): 669-683

Rowntree D. Statistics without tears: A primer for non-mathematicians: Scribner Book Company; 1981

Stølen T, Chamari K, Castagna C, Wisløff U. Physiology of soccer. Sports Med, 2005; 35(6): 501-536

Svensson M, Drust B. Testing soccer players. J Sport Sci, 2005; 23(6): 601-618

Varley MC, Aughey RJ. Acceleration profiles in elite Australian soccer. Int J Sport Med, 2013; 34(1): 34-39

Varley MC, Fairweather IH, Aughey I, Robert J. Validity and reliability of GPS for measuring instantaneous velocity during acceleration, deceleration, and constant motion. J Sport Sci, 2012; 30(2): 121-127

Zagatto AM, Beck WR, Gobatto CA. Validity of the running anaerobic sprint test for assessing anaerobic power and predicting short-distance performances. J Strength Cond Res, 2009; 23(6): 1820-1827

\section{Corresponding author:}

\section{Prof. Paulo Eduardo Redkva}

Department of Physical Education, Federal University of Parana (UFPR). Rua Coração de Maria, 92,

Jardim Botânico, Curitiba - Paraná - Brazil. CEP 80215-370.

Phone.: +55 42 9124-0048.

E-mail: pauloredkva@gmail.com 